\title{
Stability measurements of Rutherford cables with various treatments
}

\author{
S. W. Kim and D. Leroy, \\ CERN, CH-1211,Geneva, Switzerland \\ M. N. Wilson, \\ Oxford Instruments, Abingdon, Oxford, UK \\ A. K. Ghosh and W. B. Sampson, \\ Brookhaven National Laboratory, Upton, NY USA
}

\begin{abstract}
To improve the stability of Rutherford type superconducting cables, various methods of treating cables were considered and tested. These treatments include strand coating, partial soldering, "barber-pole" insulation and "porous metal" solder filling. To study the effect of these treatments, several cables were prepared and MQE (Minimum Quench Energy) values were measured. Coating, partial soldering or "barberpole insulation" improved the relative stability for some cables. The "porous metal" filled cables showed higher MQE. However the results suggest that the porous metal process needs to be improved. The measured data for cables with various treatments are presented.
\end{abstract}

\section{INTRODUCTION}

We can use a critical current (Ic) as the first indication of the stability of a superconducting cable. The critical current is a property of a strand or a cable, and this value does not change much under typical measuring conditions. However, since all superconducting cables normally operate below the critical current, we need an indication of the stability when the current is less than the critical value. The MQE (Minimum Quench Energy) may be used as this second indication.

$M Q E$ is defined as the minimum energy of a disturbance which quenches the cable or strand. This minimum energy is dependent on the cooling conditions, and should, therefore, be measured for a variety of cooling conditions. The MQE is also affected by the distribution of the disturbance in time and space, the so called "disturbance spectrum" [1].

In spite of these complications, MQE is used to characterize the stability, because it is the only way of measuring the stability when the current is less than the critical current value. Among the disturbance spectrum regions, the "short duration and small volume" region was chosen as the standard for MQE measurements. This choice was made for the following reasons [2],[3].

(1) Measurements on magnets show the duration of a spike is shorter than $100 \mu \mathrm{s}$.

(2) MQE is nearly independent of heating time, within the range between 20 and $100 \mu$ s.

Manuscript received September 14, 1998.
If one cable has a higher MQE values than another cable under this condition, it is reasonable to assume that the cable has higher MQE values also for other disturbance conditions. In a somewhat arbitrary way, the standard duration of the disturbance pulse was fixed as $45 \mu \mathrm{s}$.

The cooling of a cable is determined in part by the cable itself, and in part by the sample holder and cryostat. The cooling of the cable itself involves the surface condition, contact condition among strands, width and depth of grooves between strands and wrapping condition. We can assume these conditions to be intrinsic properties of the cable.

To compare MQE's of different cables, it is necessary to measure them with the same sample holder and cryostat. To improve the stability of a given cable, we have to make the cooling conditions better. To improve cooling, we can increase the amount of liquid helium in contact with the cable, or increase the cooling surface.

The "barber-pole insulation" scheme was considered as a way to increase the amount of liquid helium in contact [2], and this scheme improved the stability of some sample cables. To achieve further improvement, "porous metal filling" was tried to increase the cooling surface [4], and several experiments for MQE of porous metal cables were done at CERN and BNL [2],[3]. The porous metal filling was the most promising method among the methods tried.

At first, all the porous metal cable samples were made by hand. However a fabrication method was also developed to make porous metal cables, several hundreds meters long as required for LHC dipole magnets [5].

TABLE I

SPECIFICATIONS OF THE SAMPLE CABLES

\begin{tabular}{cccc}
\hline Sample ID & A & B & C \\
\hline $\mathrm{I}_{\mathrm{c}}[\mathrm{KA}]$ at $4.5 \mathrm{~K}^{\mathrm{a}}$ & 13.1 & 14.0 & 14.1 \\
$\mathrm{I}_{\mathrm{c}}[\mathrm{KA}]$ at $1.9 \mathrm{~K}^{\mathrm{a}}$ & - & - & 18.2 \\
Thickness of stainless steel core & - & - & $12 \mu \mathrm{m}$ \\
Strand diameter & \multicolumn{3}{c}{$\begin{array}{c}\text { Rutherford type cable for LHC dipole } \\
\text { No. of strands } \\
\text { Description }\end{array}$} \\
& \multicolumn{3}{c}{ inner magnet } \\
\hline
\end{tabular}

The definition of Ic is "the current when the resistivity is $10^{-14} \Omega \mathrm{m}$."

${ }^{a}$ The Ic values in this table are measured values. 
This paper describes MQE's of several Rutherford cables for LHC dipole magnets, with various treatments. The treatments include "barber-pole insulation", partial soldering, manual porous metal filling and automated porous metal filling, as mentioned above. The measurements reported here were performed in the same sample holder and cryostat at BNL. Table I shows the specifications of the three cable samples used. These three cables are nearly identical, except for sample " $\mathrm{C}$ " which has a thin stainless steel core between the two layers of strands.

\section{TREATMENTS AND WRAPPING}

Partial soldering means soldering only on the contact area between strands. This soldering makes the electrical and thermal contact resistances between strands small. Possibly, the amount of the helium inside the cable decreases, but still liquid helium can penetrate the cable.

Usually, a Rutherford cable is wrapped with an insulating tape. This insulation consists of two layers; inner layer with "double overlap" wrapping and outer layer with half spacing (like a barber pole). To increase the amount of helium contacting the surface of cable, we made the barber pole wrapping inside and the double overlap wrapping outside. This is the so called "barber-pole insulation."

The third and fourth treatments are porous metal filling in grooves of a cable. First, copper powder is mixed with soldering lead paste and then this mixture is forced into the grooves of the cable. After heating and cooling, copper particles are bonded on the surface of strands [5].

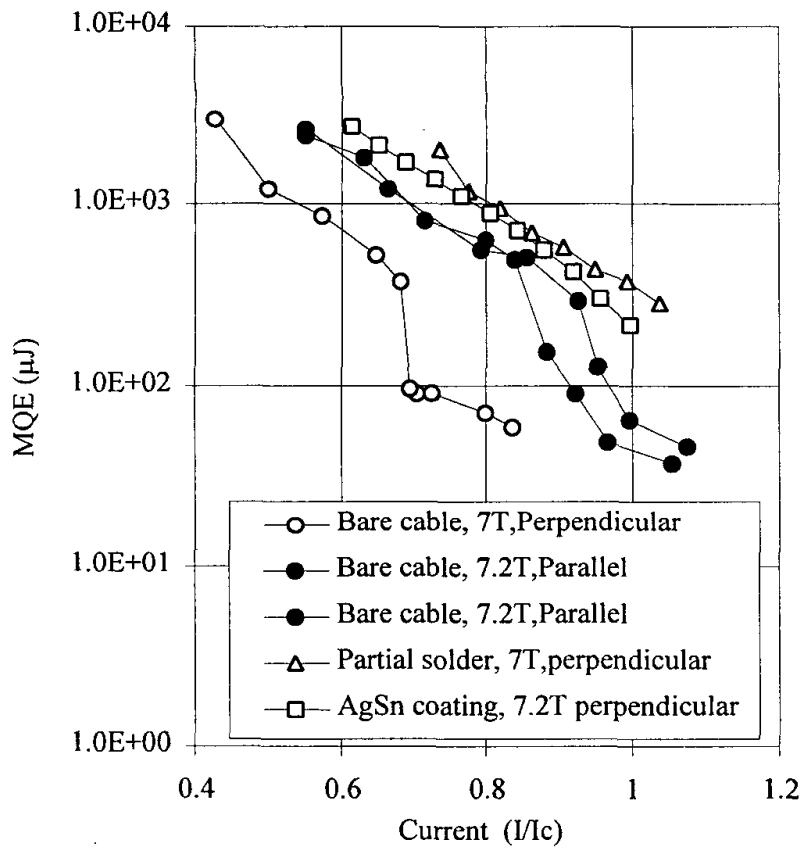

Fig. 1. Measured MQE data for sample $A$. The bath temperature was $4.5 \mathrm{~K}$

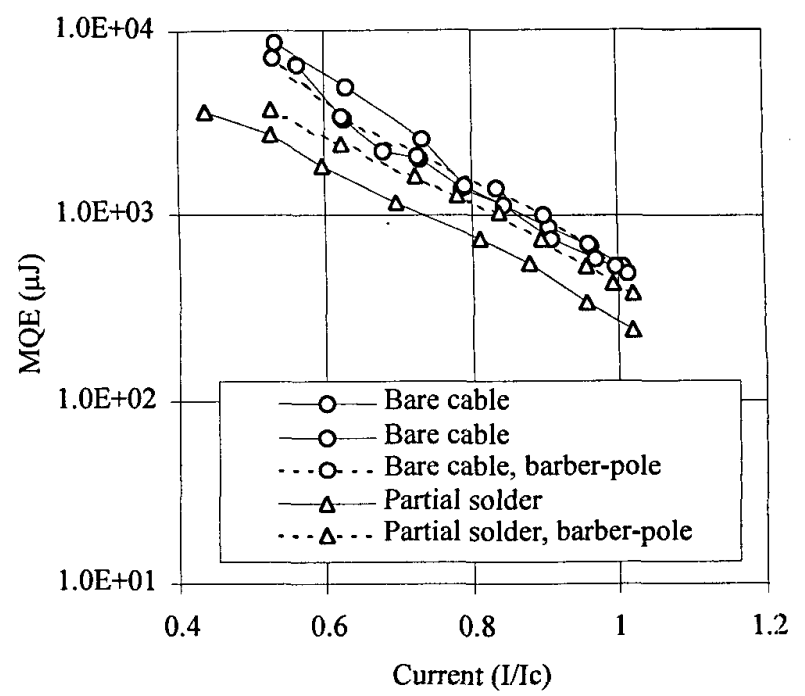

Fig. 2. Measured MQE's of the cable sample B. The bath temperature was $4.5 \mathrm{~K}$, and the magnetic field is $7 \mathrm{~T}$ parallel.

\section{EFFECT OF INSULATION AND SOLDERING}

Fig. 1 shows the measured MQE curves for sample "A". As shown in the figure, both partial soldering and $\mathrm{AgSn}$ coating increased the MQE value in the case of a perpendicular field arrangement [2],[7]. Also we can see from this figure that the MQE values in a parallel field are higher than in a perpendicular field. Possibly, this difference is caused by the field distribution, but as yet not been fully understood [7].

This figure also shows the typical "jumps" (also called "kinks"). Cables without soldering or coating have "jumps," but it seems that soldering or coating eliminates this behavior.

Fig. 2 shows the results of sample "B" in a parallel field. This sample does not present a jump in a parallel field. The cable with partial soldering shows lower MQE values. MQE of the barber-pole insulation seems to be the same value with not-treated cables. When the cable is partially soldered and insulated in barber-pole type, the MQE value becomes "lower than bare cable, but higher than partial soldering."

Fig. 3 shows the MQE's of bare and partially soldered cable sample "C". The MQE's in a parallel field are larger than those in the perpendicular orientation, but the difference between the two field orientations is no more than the difference shown in Fig. 1. As we can see in this figure, the two results in a perpendicular field are very close. We conclude from these measurements that partial soldering did not make a remarkable difference in the MQE values of cable sample " $C$ ", which has a stainless steel core. One point worth noting is that the curves in this figure show "jumps." 


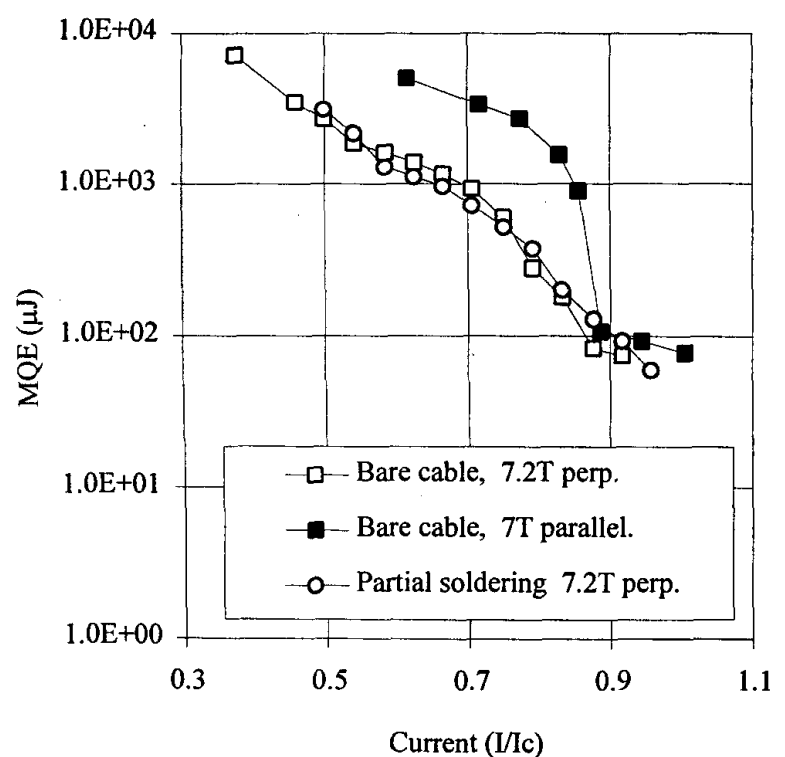

Fig. 3. MQE data for cable sample $\mathrm{C}$. This cable has a $12 \mu \mathrm{m}$ stainless steel core between two layers of the strands. The bath temperature was $4.5 \mathrm{~K}$.

MQE values of the three cables with and without coating, soldering and barber-pole insulation have been presented. Not all of the possible combinations were tested. The conclusions to date are summarized in Table II, and we conclude that these treatments do not have an appreciable effect on stability.

\section{RESULTS FOR POROUS METAL CABLES}

As mentioned above, the porous metal filling technique was developed to improve cooling of the cable, and several porous metal cables were made of cable sample " $B$ " by hand. Fig. 4 shows the MQE values of these hand-made porous metal cables. The first two series are MQE values for bare "B" cable (also shown in Fig. 2).

This figure shows that all porous metal cables have higher MQE values than bare cables. However, it is premature to speculate whether porous metal improves stability in all circumstances.

TABLE II

EFFECTS OF COATING, BARBER-POLE INSULATION AND PARTIAL SOLDERING

\begin{tabular}{cccc}
\hline Sample ID & A & B & C \\
\hline AgSn coating & better & $?$ & $?$ \\
Barber pole on bare cable & $?$ & no effect & $?$ \\
Partially soldering & better & worse & no effect \\
\hline
\end{tabular}

a In Fig. 2 the MQE values of the cable with partial solder and barber-pole are larger than those of the cable with only partial solder. However, it is not an improvement, because the values are under the MQE values of the bare cable.

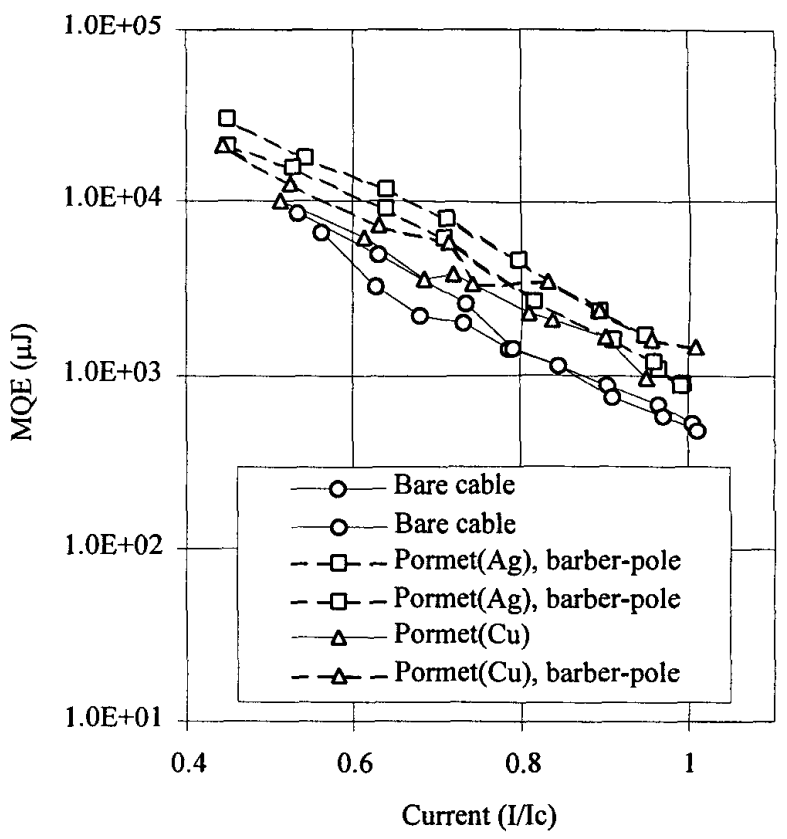

Fig. 4. MQE values of the hand made porous metal cables made of cable sample "B." The bath temperature was $4.5 \mathrm{~K}$, and field was $7 \mathrm{~T}$ parallel.

Therefore, we needed to measure another cable filled with porous metal. Also, we need "quality control" of the samples, for a fair and clear comparison. Of course, we need to measure cables that can be used for a real dipole magnet as mentioned in introduction of this paper.

We developed a process of filling "porous metal" in the grooves of a Rutherford type cable. The first stage of developing the process is described in [5], and several porous metal cable samples were made of the "C" cable. In filling porous metal, two kinds of porous metal paste were used; one was with fine copper powder and the other was with coarse powder [5].

Fig. 5 shows the result of cable sample " $C$ " with coarse powder in only the outer grooves. This cable sample labeled NICO (Nothing In Coarse Out) is the first porous metal cable produced in long length.

The curves in Fig. 5 were obtained by pulsing several heaters at different positions on each sample. There are "jumps" in the curves for bare cable and NICO. Also, we see that NICO shows a large spread in MQE, which may have been influenced by the opening of the cable during the porous metal filling process. In the filling process, a cable is twisted and opened to fill porous metal paste [5]. After filling paste, the cable is heated to make bonding. If we make this bonding more uniform, the MQE curve should show less spread in data. Anyway, Fig. 5 showed that an MQE curve of NICO can be higher than that of a cable without treatment. This suggests that we need to improve the porous metal filling process to make a more uniform and better bonding. 


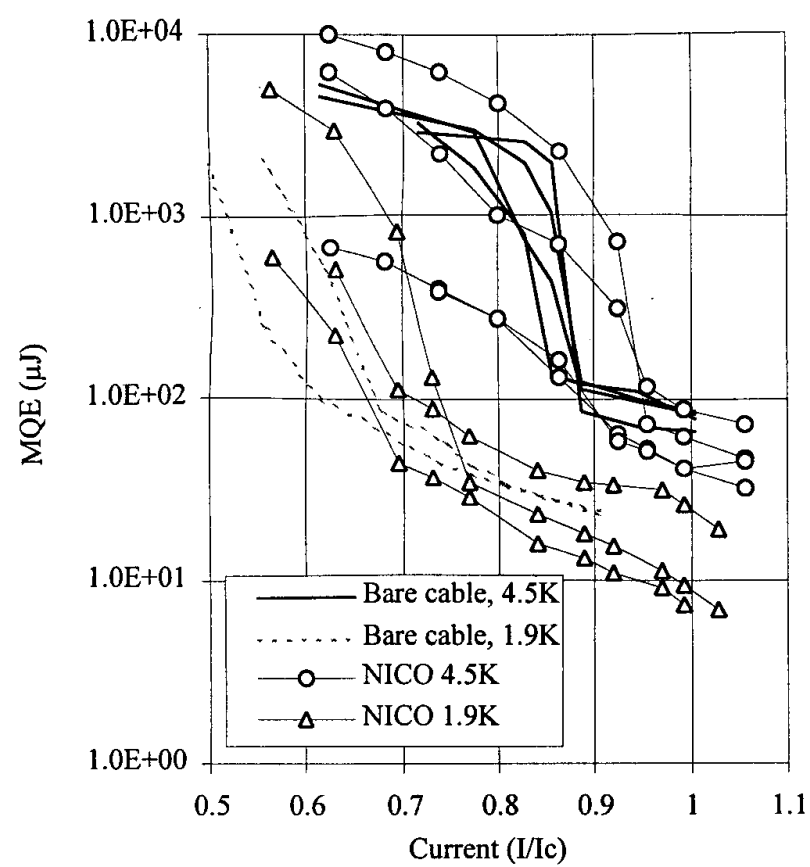

Fig. 5. Measured MQE values of NICO sample for several heaters attached on the surface. Measurements were done at $4.5 \mathrm{~K}$ and $1.9 \mathrm{~K}$, and field was $7.2 \mathrm{~T}$ parallel at $4.5 \mathrm{~K}$ and $8.7 \mathrm{~T}$ at $1.9 \mathrm{~K}$.

\section{DISCUSSIONS FOR FURTHER STUDY}

Further studies are being made in an effort to understand how to make more stable cables. We have studied some parameters. It has been recognized that the MQE values depend on the electrical and thermal resistances between the strands of the cable, the volume of helium in contact, the heat transfer coefficient and the condition of the wet surface of the strands [2],[3],[6],[7]. We have nevertheless many unsolved problems. Two of these are "the effect of the field orientation" and "precise reasons and a detailed modeling of the jumps."

Figs. 1 to 4 showed that MQE in a parallel field is higher than that in perpendicular field. We speculate that the difference is caused by the field distribution since perpendicular and parallel external fields make different field patterns in the cable. A perpendicular field arrangement gives a lower but more uniform field pattern around the heater, and in a parallel field arrangement the peak field is on the heater [2]. Several experiments were done to see the effect of field, but the results are not yet conclusive.

Figs.1, 3 and 5 showed jumps. The sample $A$ and $C$ have jumps, but not sample B. This suggests that the jumps are affected by the intrinsic properties of the cable. The existence of a core may be one of the more important properties.

An explanation for the jumps has been proposed by Wilson. That was the most possible explanation, which said that at a jump, the cable is switching between the single strand behavior and the collective behavior [2]. However, the model needs to be improved to answer to the following questions:

- A jump appears in a wide range of current $(0.7 \mathrm{Ic} \sim 0.95 \mathrm{Ic}$ in Fig. 5). When the current is 0.7Ic for instance, there should be enough capacity for current. How a one-strandquench can then quench the whole cable?

- Some cables showed two jumps in a curve (Figs. 3 and 5).

- Some cables didn't show a jump (Figs. 2 and 4). Did they show only the strand behavior, only the cable behavior or an extremely smooth switching?

- In a logarithmic scale, an MQE curve of a cable and that of a strand are in parallel in the high current region [2], [3],[6]. We thought that is the strand behavior. In a linear scale, the MQE curves of cable and strand show nevertheless different slopes.

All these questions suggest that we need a new model for the electro-thermal dynamical behavior of a Rutherford cable.

\section{CONCLUSIONS}

Several kinds of Rutherford cables for LHC dipole with various treatments were tested. From the measurement we conclude the following.

1. Coating, partial soldering and barber-pole insulation do not always lead to an improvement in stability.

2. Porous metal filling is a promising method to increase stability, but the porous metal filling process needs to be improved.

3. MQE is also affected by field orientation, further study is needed to understand it.

4. Cables with cores showed "jumps" and the stability was rather variable from heater to heater location. Further study is needed, also.

\section{REFERENCES}

[1] S. L. Wipf and A. P. Martinelli, Proc. 1972 Applied Superconductivity Conference, Annapolis, IEEE 72CH0682-5-TABSC. pp331.

[2] M. N. Wilson, W. B. Sampson and A. K. Ghosh, "Experimentally measured Minimum Quench Energies of LHC cables," LHC project report 86, CERN, Jan 1997.

[3] A. K. Ghosh, W. B. Sampson and M. N. Wilson, "Minimum Quench Energies of Rutherford Cables and Single Wires," IEEE Trans. on Applied Superconductivity, vol. 7(2), pp954-957 (1997).

[4] D. E. Baynham, V. W. Edwards and M. W. Dean, "Transient stability enhancement in high current density cables," Workshop on refrigeration science and technology, Paris, 1981.

[5] Seog-Whan Kim and John Dave Adam, "Fabrication of porous metal cables," LHC-MMS internal note 98-02, CERN, Jan 1998.

[6] A.K. Ghosh, W.B. Sampson, S.W. Kim, D. Leroy, L.R. Oberli and M.N. Wilson, "Stability Measurements on Cored Cables in Normal and Superfluid Helium," International Cryogenics Material Conference 98, Enschede, Netherlands, May 10-13, 1998.

[7] S. W. Kim, M. N. Wilson, A. K. Ghosh and W. B. Sampson, "Experiments on Minimum Quench Energy of LHC cables with various treatment," LHC-MMS internal note, CERN, 1998, to be published.

[8] A. Kimura, S. W. Kim, N. Kimura, Y. Makida, T. Shintomi, H. Hirabayashi, T. Mito, A. Iwamoto, J. Yamamoto, "Stabilities of the Rutherford Cables with $\mathrm{Cu}$ and CuMn Barrier," IEEE Trans. on Applied Superconductivity, Vol. 5, No. 2, pp 385-388, June 1995. 